\title{
Unevenly distributed LED light produces distinct behavioral preferences and production performance of broilers
}

\author{
Chenghao Pan ${ }^{1}$, Minsi Lu ${ }^{1}$, Yingping Zhang ${ }^{2}$, Yue Yu ${ }^{1}$, Qiuyan Lu$^{3}$, Yefeng Yang ${ }^{1}$, Jinming Pan $^{1 *}$ \\ (1. College of Biosystems Engineering \& Food Science, Zhejiang University, Hangzhou 310058, China; \\ 2. College of Optical and Electronic Technology, China Jiliang University, Hangzhou 310018, China; \\ 3. Sino-Agri Leading Biosciences Company, Ltd, Shanghai 200071, China)
}

\begin{abstract}
LED has shown great advantages in poultry husbandry. This study focused on the behavioral preferences and production performance of chicken broilers reared under unevenly distributed yellow LED light. Four pens were divided into two groups adopting respective maximum light intensities (MLIs, $60 \mathrm{~lx}$ and $30 \mathrm{~lx}$ ). Because of different distances from the installation position of the LED pipe, each pen was distributed with unevenly distributed light. Each pen consisted of four subzones indicated by their light intensities-high intensity (HI), medium intensity (MI), low intensity (LI) and weak intensity (WI). Four subzones were the same size and provided with a feeder and a drinker, respectively. The broilers moved freely across the four subzones. No significant differences in body weight (BW), uniformity of final BW and feed conversion ratio (FCR) were observed between the two experimental groups. However, greater feed intake and water intake were found in HI than those in other subzones. The drinking preference changed with age for four subzones and was more likely to appear at the later stage, despite substantial fluctuations within the replicates. The feeding preference was more constant than the drinking preference and appeared mainly at the early and middle stages of this study. These findings could provide implications for broiler production reared under unevenly distributed LED light condition.
\end{abstract}

Keywords: LED, unevenly distributed light, behavioral preference, broiler production performance, light intensity, artificial lighting, animal welfare

DOI: $10.25165 /$ j.ijabe.20191202.4689

Citation: Pan C H, Lu M S, Zhang Y P, Yu Y, Lu Q Y, Yang Y F, et al. Unevenly distributed LED light produces distinct behavioral preferences and production performance of broilers. Int J Agric \& Biol Eng, 2019; 12(2): 49-53.

\section{Introduction}

Fowls have a more refined visual system than that of humans, indicating that fouls may perceive light that is imperceptible for humans ${ }^{[1,2]}$. Previous studies reported the influences of light management on broilers, including factors such as body weight (BW), BW gain, feed intake, feed conversion ratio (FCR), and welfare $^{[3-5]}$. Among light intensity, wavelength spectrum and photoperiod duration, light intensity is usually considered to have the greatest association with behaviors and activities. Therefore, on commercial farms, light intensity is commonly used as an important tool in the manipulation of light environment. Housing under high light intensity (200 lx) might improve the welfare of broilers $^{[6]}$. What is more, higher light intensity might improve skeletal health by stimulating activities, further improving the welfare of the birds ${ }^{[6-8]}$. Broiler behavioral preferences were also evident under particular light intensities, depending on the rearing

Received date: 2018-10-08 Accepted date: 2019-03-09

Biographies: Chenghao Pan, Master candidate, research interests: spectral sensitivity of poultry, Email: 21713013@zju.edu.cn; Minsi Lu, Master, research interests: animal visual perception, Email: lvminsi@126.com; Yingping Zhang, Master, Lecturer, research interests: animal light environment, Email: zhangyingping@cjlu.edu.cn; Yue Yu, PhD, Postdoctor, research interests: animal light environment, Email: yuyueoffice2012@126.com; Qiuyan Liu, Bachelor, Engineer, research interests: animal light environment, Email: lqy860618@126.com; Yefeng Yang, PhD, Postdoctor, research interests: animal visual perception, Email: yangyefeng_0920@163.com;

*Corresponding author: Jinming Pan, PhD, Professor, research interests: animal light environment, College of Biosystems Engineering \& Food Science, Zhejiang University, Hangzhou 310058, China. Tel: +86-571-88982282, Email: panhouse@zju.edu.cn. phase $\mathrm{e}^{[9,10]}$. By contrast, dim light $(<10 \mathrm{~lx})$ resulted in increased eye weight ${ }^{[7,8,11]}$, increased incidence of skeletal disorders ${ }^{[11,12]}$ and decreased activity ${ }^{[4,8]}$. Despite many negative effects on broiler production and welfare, most broilers in modern commercial facilities are reared under low light intensities $(<5 \mathrm{~lx})$ to improve FCR and reduce carcass damage ${ }^{[11]}$. The light environment in broiler production facilities must be strictly managed to meet the physiological needs of broilers ${ }^{[13]}$, but there is a debate regarding the appropriate level that required in the light management ${ }^{[8,11]}$.

Artificial lighting is widely used on commercial farms. It was observed that broilers presented better production performance under LED lighting than broilers under compact fluorescent lamps ${ }^{[14]}$. The BW and blood platelet count of broilers were numerically higher in yellow LED light than those in incandescent light ${ }^{[15]}$. Nowadays, incandescent lamps are gradually prohibited. Meanwhile, LED light sources show a great potential in animal production facilities compared to traditional light sources. LEDs have many advantages, such as high energy efficiency, long lifetime and high monochromaticity ${ }^{[16,17]}$, which make LEDs stand out from several common light sources. Therefore, LEDs were utilized as the light source in this study. Because of different distances from the installation position of the LED pipe, the floor light field of each pen was non-uniform. Uniformity is an indicator in the evaluation of lighting quality of environment. The Min: Avg uniformity indicator, defined as the ratio of the minimum illuminance value to the average value, has been widely used in engineering ${ }^{[18]}$. Nonetheless, researches about unevenly distributed LED light and its effects on birds are extremely inadequate.

We previously performed a study about the effect of unevenly distributed yellow LED light with light intensities of $90 \mathrm{~lx}$ and 
$60 \mathrm{~lx}$ on the behavioral preferences and performance of female broilers, and concluded that broilers' behavioral preferences were collectively affected by age, time phase and light intensity ${ }^{[19]}$. The photoperiod under different light intensities was obtained based on broilers' initiative choice. However, the light intensity subzones were not identical in our previous study. Based on that previous study, the experimental setup was optimized applying different light intensities of $60 \mathrm{~lx}$ and $30 \mathrm{~lx}$, which were lower than before. The shapes of light intensity subzones were the same to eliminate the effects on broilers caused by zoning division. The purpose of this study was to further investigate whether unevenly distributed LED light with light intensities of $60 \mathrm{~lx}$ and $30 \mathrm{~lx}$ would produce distinct preferences and performance of Xiaoshan female broilers. This study might provide reference information about broilers' performance and behavior patterns under LED light environments

\section{Materials and methods}

\subsection{Pens with unevenly distributed LED light}

This study was performed on Yuhang Qinqin Poultry Farm, Hangzhou, China. As shown in Figure 1, four pens $(2 \mathrm{~m} \times 0.5 \mathrm{~m}$ $\times 0.5 \mathrm{~m}$, length $\times$ width $\times$ height) were built to provide 2 non-uniform light fields with respective maximum light intensities (MLIs) of $60 \mathrm{~lx}$ and $30 \mathrm{~lx}$ in two replicates. The MLI of the light field was measured in the center of the right or left pen side (Figure 1a). Each pen was $0.5 \mathrm{~m}$ above the ground to improve the ventilation and was surrounded by a $0.5 \mathrm{~m}$ high iron wire net barrier to prevent the broilers from escaping. Each subzone was furnished with a set of feeder and drinker attached outside the pen (Figure 1a).

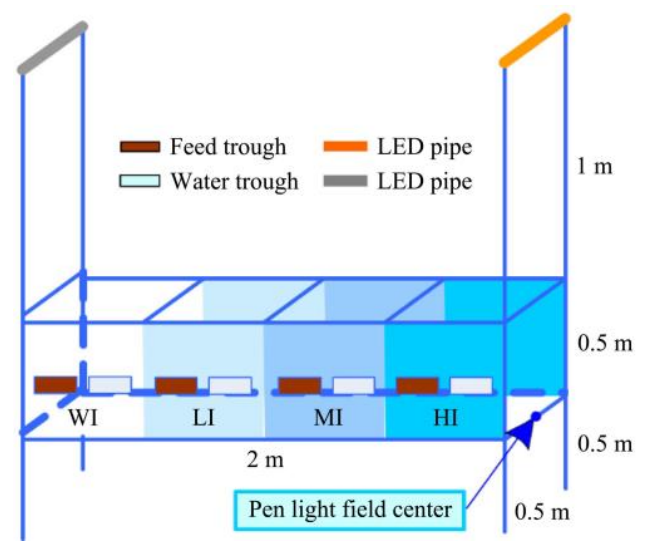

a. Installation of LED pipes

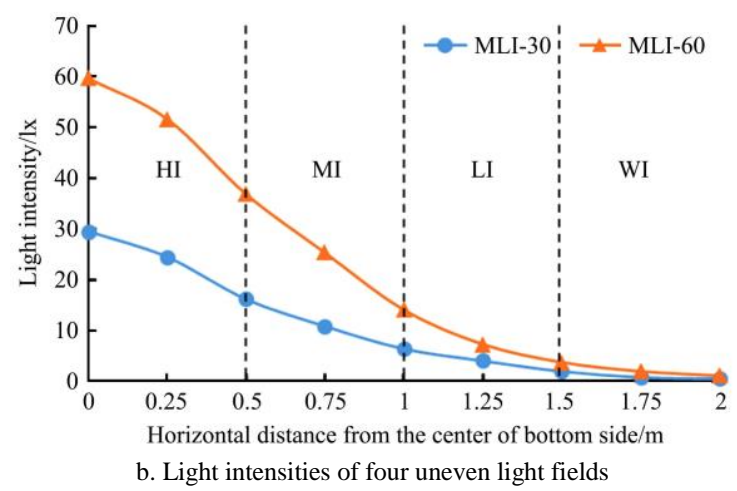

Note: Two LED pipes were on alternately for a 7-day cycle. Yellow indicated on, and gray indicated off. The light intensity distribution was measured from the pen light field center. HI, MI, LI and WI: high intensity, medium intensity, low intensity and weak intensity subzones, respectively.

Figure 1 Layout of pens
In each pen, two 60-cm long yellow LED pipes (model: LTBB52; peak wavelength: $591.1 \mathrm{~nm}$; half wave width: $16 \mathrm{~nm}$; rated power: $4.32 \mathrm{~W}$; vendor: Langtuo Biotechnology Co., Hangzhou, China) were installed at a height of $2.0 \mathrm{~m}$ above the ground (Figure 1a). Two levels of MLIs, 60 lx for Group MLI-60 and $30 \mathrm{~lx}$ for Group MLI-30, were obtained by regulating the voltage of LED pipes with a pulse width modulation controller. The light field of each pen was non-uniform and subsequently divided into four $0.5 \mathrm{~m} \times 0.5 \mathrm{~m}$ subzones, indicated by their light intensities-high intensity (HI), medium intensity (MI), low intensity (LI) and weak intensity (WI). The light intensity distribution, measured with a light meter (AR823; Shuangxu Electronics Co., Ltd., Shanghai, China), showed a smooth decrease as a function of the horizontal distance from the LED pipe (Figure 1b). The LED pipes were on alternately for a 7-day cycle to prevent the broilers from acclimating to the environment and to further exclude interference from the environment. The lighting regime in our study was set to 16L: $8 \mathrm{D}$ (16 h light: $8 \mathrm{~h}$ dark per day) with which broilers had a better welfare status ${ }^{[20]}$.

There are several types of indicators used for the evaluation of uniformity of light. The Min: Avg uniformity indicator, defined as the ratio of the minimum illuminance value to the average value, is simple and intuitive ${ }^{[18]}$. It is calculated using the Equation (1):

$$
\operatorname{Min}: A v g=\frac{E_{\min }}{E_{\text {avg }}}
$$

where, $E_{\min }$ is the minimum illuminance of non-uniform lighting, $1 \mathrm{x}$; and $E_{\text {avg }}$ is the average illuminance of non-uniform lighting, lx. Generally, the uniformity of light designed for people in indoor lighting is greater than 0.7. Two ratios were 0.037 for Group MLI-60 and 0.036 for Group MLI-30.

\subsection{Management of broilers}

A total of 20 Xiaoshan female broilers (after brooding) were obtained from the Zhejiang Guangda Breeding Poultry Corporation (Jiaxing, China). These birds were reared under incandescent lamps for the first four weeks. Then the 20 birds were randomly distributed into 4 pens and tested for 8 weeks in Group MLI-60 or Group MLI-30. Each light treatment had two replicates, with 5 broilers per pen. The birds were allowed to move freely according to their preferences for the unevenly distributed yellow LED light. Each set of feeder and drinker was specifically supplied with adequate food and water every day to avoid competition of the broilers. The heights of the feeders and drinkers above the pen floor were adjusted according to the age of broilers. The ambient temperatures in the pens were $33^{\circ} \mathrm{C} \pm 2^{\circ} \mathrm{C}$ in the first two weeks and $22^{\circ} \mathrm{C} \pm 1^{\circ} \mathrm{C}$ in the following period.

\subsection{Measurements and data analyses}

The added and remaining food in four subzones were recorded daily and summarized weekly (weeks 5-11). Besides, the water intake per bird in four subzones were recorded every Saturday (weeks 5-11). Moreover, the broilers were weighed every 2 weeks (weeks 6, 8, 10 and 12). The tested indexes obtained from the measurements were feed intake, water intake, FCR, mortality and uniformity $[=(1-$ standard deviation/mean $) \times 100 \%]$ of final BW. The feeding and drinking preferences in four subzones were indicated by feed intake and water intake respectively. The data was examined for the significance of differences by analysis of variance using IBM SPSS Statistics 22. Means and significant interactions were compared with a Duncan's Multiple Range Test ${ }^{[7]}$ at $p<0.05$ level. 


\section{Results and discussion}

\subsection{Performance characteristics}

During days 28 to 84 , production performance parameters, including BW, FCR, and feed intake, were not significantly affected by the light intensity $(p>0.05)$ (Table 1$)$. These effects were in agreement with most previous researches. In general, light intensities higher than $1 \mathrm{~lx}$ had no obvious effect on BW, food intake, and $\mathrm{FCR}^{[11,21,22]}$. However, the findings of recent researches contradicted that increasing illuminance caused a decline in $\mathrm{BW}^{[8,23,24]}$. It was reported that housing under low light intensities decreased activity to promote fat accumulation and induce BW to gain ${ }^{[8,25]}$. Furthermore, light intensity was often kept low (generally below $5 \mathrm{~lx}$ ) in practical production to inhibit bird activity ${ }^{[4,8,25]}$ and save energy. It was unclear whether genetics or facilities led to distinct effects on performance under different light intensities. No broiler died during the study, suggesting that mortality was not affected by the varying light intensity from $30 \mathrm{~lx}$ to $60 \mathrm{~lx}$ under unevenly distributed LED light environments. It also provided the basis for the application of LED lights.

Table 1 Effects of uneven yellow LED light with respective maximum light intensities (MLIs) on body weight (BW), final BW, uniformity, feed intake, feed conversion ratio (FCR), and mortality of female broilers during days 28 to 84

\begin{tabular}{ccccccc}
\hline \multirow{6}{*}{$\begin{array}{c}\text { MLI } \\
/ \mathrm{lx}\end{array}$} & $\begin{array}{c}\text { Initial } \\
\text { BW/g }\end{array}$ & $\begin{array}{c}\text { Final BW } \\
/ \mathrm{g}\end{array}$ & $\begin{array}{c}\text { Uniformity } \\
/ \%\end{array}$ & $\begin{array}{c}\text { Feed } \\
\text { intake/kg }\end{array}$ & $\begin{array}{c}\text { FCR } \\
/ \mathrm{g} \cdot \mathrm{g}^{-1}\end{array}$ & $\begin{array}{c}\text { Mortality } \\
/ \%\end{array}$ \\
\hline 60 & $393.2 \pm 0.2$ & $1411.2 \pm 15.5$ & $94.8 \pm 2.5$ & $3.21 \pm 0.01$ & $3.15 \pm 0.04$ & 0 \\
30 & $389.7 \pm 9.9$ & $1379.9 \pm 42.4$ & $95.0 \pm 1.0$ & $3.51 \pm 0.43$ & $3.50 \pm 0.32$ & 0 \\
\hline
\end{tabular}

Note: Values are means \pm SE. Values do not differ significantly $(p<0.05)$. The birds were not assigned to replicates before $28 \mathrm{~d}$ of age. Initial $\mathrm{BW}$, final $\mathrm{BW}$ and feed intake were gained from the average value of five broilers in one replicate. Uniformity was obtained from the final BW of five broilers in each pen.

It was concluded that two light intensities, MLI-60 and MLI-30, had no influence on BW gain in different growing stage of broilers $(p>0.05)$, which was shown in Figure 2. A possible cause may be related to higher light intensity in our study compared with practical production. Although BW gain had a great increase during weeks 7-8, FCR did not fluctuate significantly in Group MLI-60 and Group MLI-30 during weeks 5-10. Maybe light intensity did not affect FCR during the transition from breeding period to fattening period. However, a significant increase was found for FCR in previous researches ${ }^{[26]}$.

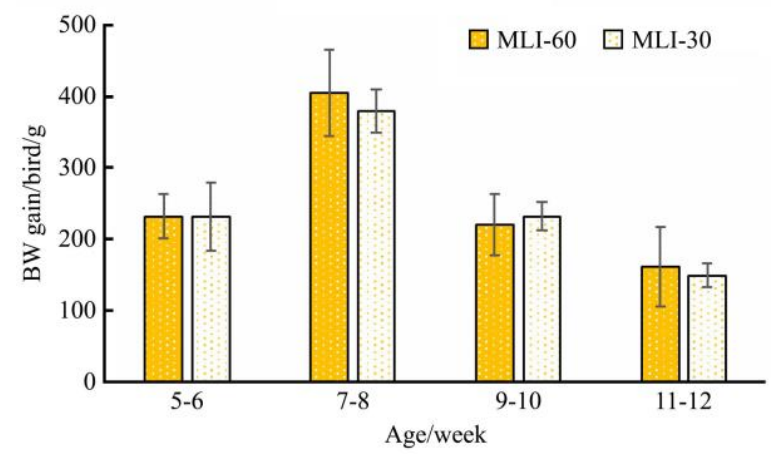

Figure 2 BW gain of broilers every two weeks in different lighting groups during weeks 5-12

3.2 Drinking preferences in groups with different maximum light intensities

In this study, the water intake per bird in four subzones were recorded every Saturday as the indicator of subzone preference for drinking behavior. MLI-60-H, MLI-60-M, MLI-60-L and MLI-60-W indicate the high-intensity, medium-intensity, low-intensity, and weak-intensity subzones, respectively, in Group MLI-60. The subzones in Group MLI-30 are labeled analogously. Throughout the experiment, a significant behavioral preference for drinking was observed in both groups. Birds preferred to drink in HI rather than in other subzones $(p<0.05)$, but no difference in water consumption was observed among MI, LI and WI ( $p>0.05)$.

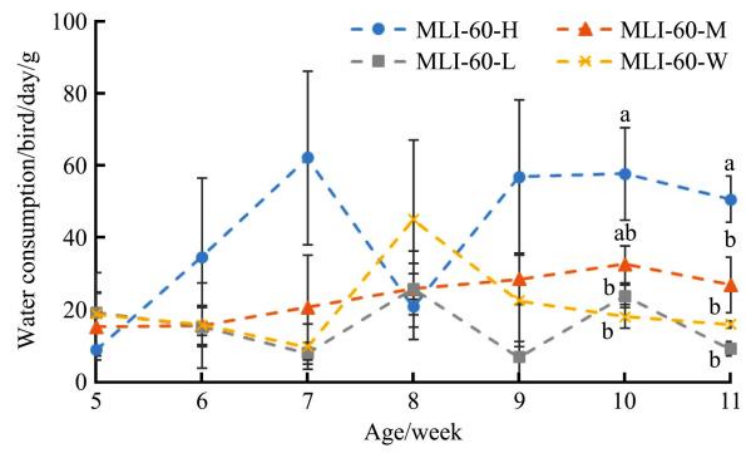

a. Daily water consumption in Group MLI-60

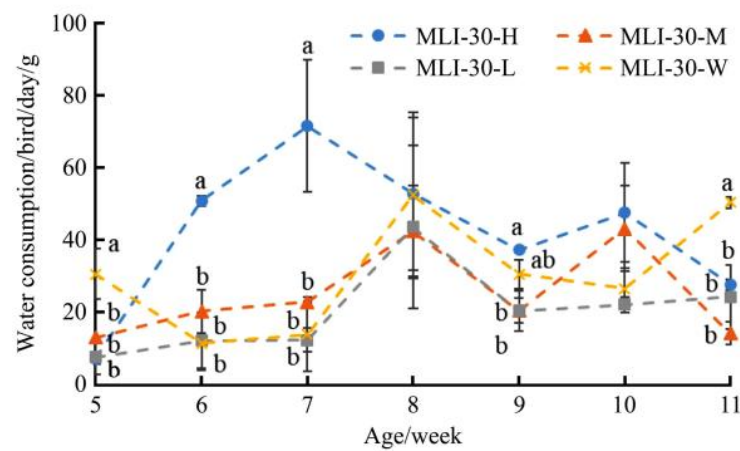

b. Daily water consumption in Group MLI-30

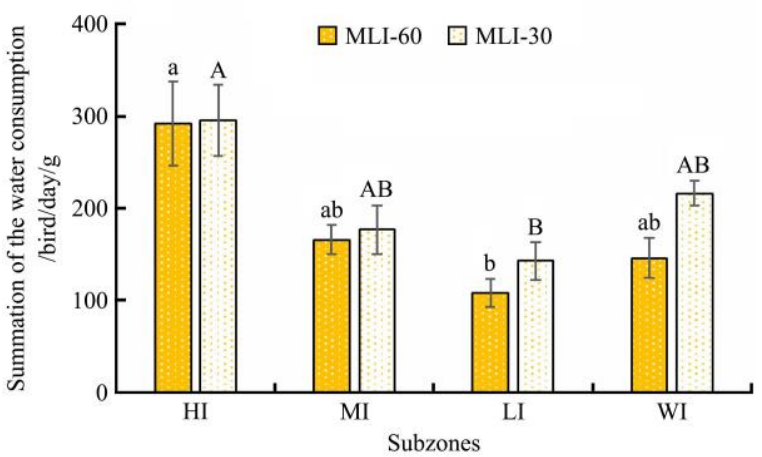

c. Summation of the daily water consumption

Note: Different letters indicate significant differences. Lowercase letters ( $a$ and $b$ ) and uppercase letters (A and B) indicate $p<0.05$.

Figure 3 Daily water consumption and summation of the daily water consumption per bird for four lighting subzones in two lighting groups during weeks 5-11

There were no consistent patterns strictly in both groups. In Group MLI-60, the water intake in HI and MI had a significant difference, but each of them was basically greater than that in LI or WI, especially LI (Figure 3a). There were no obvious differences in four subzones $(p>0.05)$ during week 5 probably caused by not completely adaptive to the new light environment. In Group MLI-30, broilers tended to drink most in HI during weeks 6-7, but the differences between $\mathrm{HI}$ and other subzones decreased during weeks 8-11 (Figure 3b). According to the statistical analysis, the timing of drinking preference changed in both groups. In Group 
MLI-60, the drinking preference appeared at weeks 10 and 11, and the water intake in HI was significantly greater than that in LI and WI $(p<0.05)$, particularly in week $11 \quad(p<0.01)$. These results indicated that the drinking preference was more likely to appear during the later stage (weeks 10-11). Nonetheless, the broilers tended to drink most in $\mathrm{HI}$ at the early stage (weeks 5-7). However, in Group MLI-30, the drinking preference appeared in weeks 5, 6, 7, 9 and 11. The preference changed significantly in weeks 5 and 11, with the water intake in WI much greater than that in HI, whereas HI remained dominant during weeks 6,7 and 9. No drinking preference was found for week 8 in both groups ( $p>$ $0.05)$.

Although the overall water consumption of four subzones in Group MLI-30 was a little greater than that in Group MLI-60, the water intake of four subzones increased with light intensity except for WI (Figure 3c). Summation of the daily water consumption also indicated that the broilers tended to drink more in HI than in other subzones. Nevertheless, statistical analysis indicated that no linear relation was presented between the drinking preference and the light intensities. It was possible that no difference among the subzones was detected because of the masking effect of the marked fluctuations within the replicates. This outcome also indicated the inconsistency of the drinking behavior for broilers, particularly at the early stage. This result was not consistent with previous research which reported that age did not affect the drinking behavior for broilers ${ }^{[27]}$, possibly because of different illuminances of light environments.

\subsection{Feeding preferences in groups with different maximum light intensities}

The feed intake per bird every week in four subzones were the indicator of the subzone preference for feeding behavior. The feeding preference was more constant than the drinking preference. Maybe it was caused by the evaporation of water. In both groups, the feed intake in HI was still greater than that in other subzones.

The timing of the feeding preference in both groups differed insignificantly, with weeks 5, 7, and 9 in Group MLI-60 (Figure 4a) and weeks 6, 7 and 9 in Group MLI-30 (Figure 4b). No feeding preference was found in any group for week $8(p>0.05)$, whereas there was an obvious increase of the feed intake. The feeding preference appeared mainly at the early and middle stages (weeks 8-9) of growth, while the drinking preference appeared primarily at the later stage of growth. In both groups, an obvious change happened in week 8 for the water intake and food intake. The overall feed consumption also showed that the feed intake in HI was much greater than that in other subzones, indicating that the feeding behavior of broilers occurred more frequently in HI during growth cycles. Specifically, the feed intake almost decreased linearly with decreased light intensity in Group MLI-60 (Figures 1b and 4c). Moreover, the overall feed consumption in MI, LI, and WI in Group MLI-30 were basically the same.

Overall, under the LED light environments of 30-60 lx during weeks 5-11, the drinking behavior of the broilers was disordered, including HI preference at the early stage, WI preference at the later stage, and no constant preference to other light intensities was observed. Although the feeding preference for $\mathrm{HI}$ appeared mainly at the early and middle stages, the feeding preference for other subzones increased at the later stage and there were no obvious differences among four subzones. The findings of present researches are consistent with previous conclusion that older birds preferred to stay in dim light ${ }^{[19]}$. The possible reason for the difference may be the complicated and multi-factor nature of poultry behavior research itself, such as behavior changes with environmental factors. It was reported that behavior preferences of birds was influenced by previous experience ${ }^{[28-30]}$ and social property $^{[31]}$. Specifically, birds previously reared in different light environment, would make different choices, and the role played as an individual or a group would lead to different results.

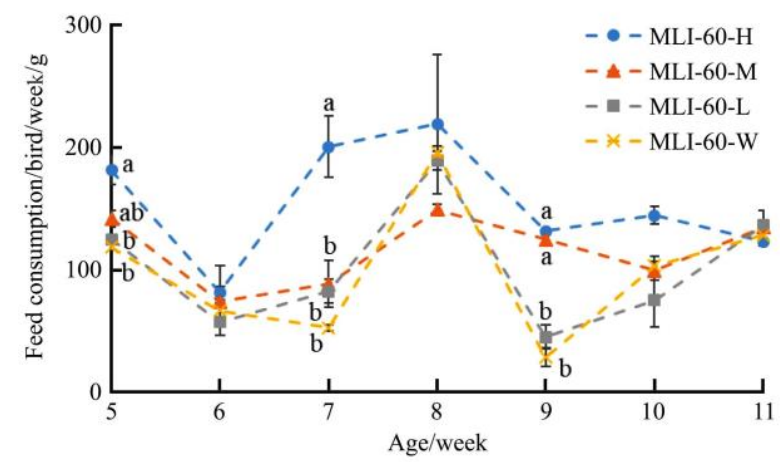

a. Weekly feed consumption in Group MLI-60

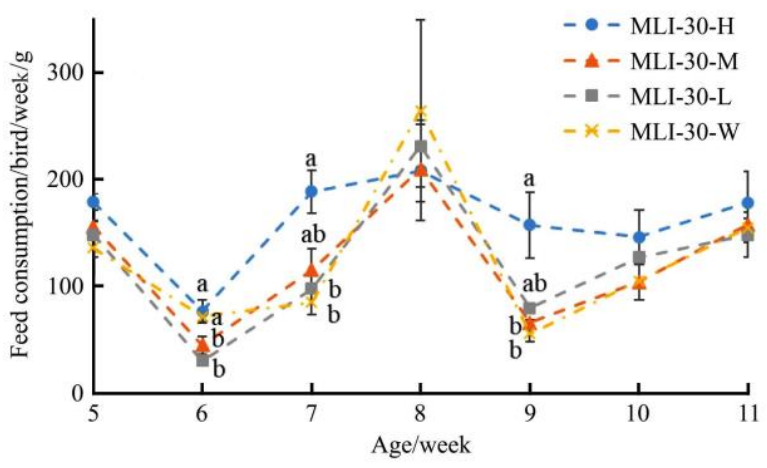

b. Weekly feed consumption in Group MLI-30

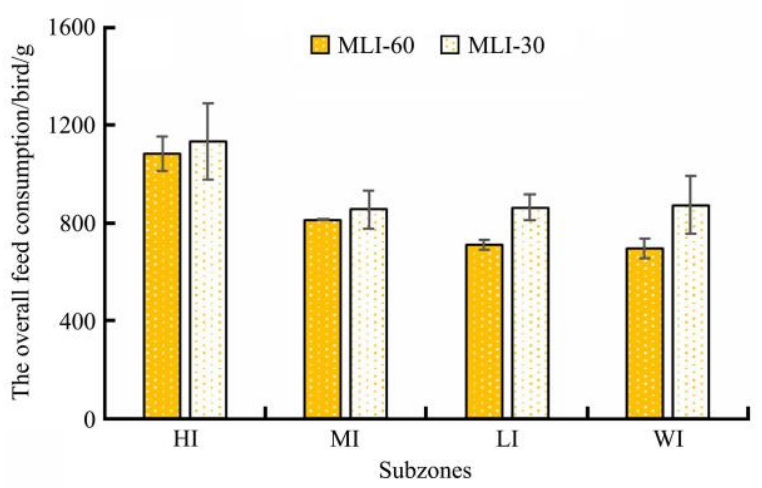

c. Overall feed consumption

Note: Different letters indicate significant differences. Lowercase letters (a and b) and uppercase letters (A and B) indicate $p<0.05$.

Figure 4 Weekly feed consumption and the overall feed consumption per bird for four lighting subzones in different lighting groups during weeks 5-11

\section{Conclusions}

It was concluded that alternativeMLIs of $30 \mathrm{~lx}$ or $60 \mathrm{~lx}$ in unevenly distributed yellow LED light environments had no significant effect on BW, BW gain, feed intake, feed and FCR of chicken broilers. However, birds preferred to drink and feed in $\mathrm{HI}$, and no constant preference was found in other subzones. In Group MLI-60, drinking preference was more likely to appear at the later stage. In Group MLI-30, the broilers tended to drink most in $\mathrm{HI}$ at the early stage, while the differences between $\mathrm{HI}$ and other subzones decreased at the later stage. The feeding preference was more constant than the drinking preference. The broilers showed a preference for feeding under the highest intensity 
light and rejection for feeding under the weakest intensity light, primarily at the early and middle growth stages.

In summary, unevenly distributed LED light produces distinct behavioral preferences and production performance differences in broilers. When apply the LED lights in broiler production, we should pay attention to the unevenly distributed light. The results of present study can be used to design light environment in practical production. Further studies will be conducted with consideration of color and intensity of LED light.

\section{Acknowledgements}

We acknowledge the support from National Key R\&D Program of China (Grant No. 2017YFB0404000), China Postdoctoral Science Foundation (2018M632470), and Postdoctoral Science Foundation of Zhejiang Province.

\section{[References]}

[1] Barber C L, Prescott N B, Jarvis J R, Sueur C L, Perry G C, Wathes C M. Comparative study of the photopic spectral sensitivity of domestic ducks (Anas platyrhynchos domesticus), turkeys (Meleagris gallopavo gallopavo) and humans. Br Poult Sci, 2006; 47(3): 365-374.

[2] Saunders J E, Jarvis J R, Wathes C M. Calculating luminous flux and lighting levels for domesticated mammals and birds. Animal, 2008; 2(6): 921-932.

[3] Lien R J, Hess J B, McKee S R, Bilgili S F, Townsend J C. Effect of light intensity and photoperiod on live performance, heterophil-to-lymphocyte ratio, and processing yields of broilers. Poult. Sci., 2007; 86(7): 1287-1293.

[4] Deep A, Schwean-Lardner K, Crowe T G, Fancher B I, Classen H L. Effect of light intensity on broiler behaviour and diurnal rhythms. Appl. Anim. Behav. Sci., 2012; 136(1): 50-56.

[5] Rogers A G, Pritchett E M, Alphin R L, Brannick E M, Benson E R. I. Evaluation of the impact of alternative light technology on male broiler chicken growth, feed conversion, and allometric characteristics. Poult. Sci., 2015; 94(3): 408-414.

[6] Alvino G M, Archer G S, Mench J A. Behavioural time budgets of broiler chickens reared in varying light intensities. Appl. Anim. Behav. Sci., 2009; 118: 54-61.

[7] Fidan E D, Nazlıgül A, Türkyılmaz M K, Aypak S Ü, Kilimci F S, Karaarslan S, et al. Effect of photoperiod length and light intensity on some welfare criteria, carcass, and meat quality characteristics in broilers. Rev. Bras. Zootec., 2017; 46(3): 202-210.

[8] Rault J L, Clark K, Groves P J, Cronin G M. Light intensity of 5 or 20 lux on broiler behavior, welfare and productivity. Poult. Sci., 2017; 96(4): 779-787.

[9] Downs K M, Lien R J, Hess J B, Bilgili S F, Dozier W A. The effects of photoperiod length, light intensity, and feed energy on growth responses and meat yield of broilers. Journal of Applied Poultry Research, 2006; 15(3): 406-416.

[10] Skrbic Z, Lukic M, Petricevic V, Bogosavljevic-Boskovic S, Tolimir N, Doskovic V, et al. Effects of intensity of light and stocking density on broiler body weight and yield of valuable carcass parts. Biotechnology in Animal Husbandry, 2018; 34(1): 83-93.

[11] Deep A, Schwean-Lardner K, Crowe T G, Fancher B I, Classen H L. Effect of light intensity on broiler production, processing characteristics, and welfare. Poult. Sci., 2010; 89(11): 2326-2333.

[12] Deep A, Raginski C, Schwean-Lardner K, Fancher B I, Classen H L. Minimum light intensity threshold to prevent negative effects on broiler production and welfare. Br Poult Sci, 2013; 54(6): 686-694.

[13] Hamrita T K, Conway R H. First order dynamics approaching of broiler chicken deep body temperature response to step changes in ambient temperature. Int J Agric \& Biol Eng, 2017; 10(4): 13-21.

[14] Mendes A S, Paixao S J, Restelatto R, Morello G M, Moura D J D, Possenti J C. Performance and preference of broiler chickens exposed to different lighting sources. The Journal of Applied Poultry Research, 2013 22(1): 62-70.

[15] Kim M J, Parvin R, Mushtaq M M, Hwangbo J, Kim J H, Na J C, et al. Growth performance and hematological traits of broiler chickens reared under assorted monochromatic light sources. Poult. Sci., 2013; 92(6): 1461-1466.

[16] Pan J, Wang X, Jiang J, Yu Y, Chen X. Advancement and trend of led light environment control technology for intensive poultry production. Transactions of the CSAM, 2013; 44(9): 225-235. (in Chinese)

[17] Song J X, Meng Q W, Du W F, He D X. Effects of light quality on growth and development of cucumber seedlings in controlled environment. Int J Agric \& Biol Eng, 2017; 10(3): 312-318.

[18] Yao Q, Zhong B T, Shi Y, Ju J Q. Evaluation of several different types of uniformity metrics and their correlation with subjective perceptions. Leukos, 2016; 13(1): 33-45.

[19] Pan J, Lu M, Lin W, Lu Z, Yu Y, Yue Y, et al. The behavioral preferences and performance of female broilers under unevenly distributed yellow LED lights with various intensities. Transactions of the ASABE, 2014; 57(4): 1245-1254.

[20] Bayram A, Ozkan S. Effects of a 16-hour light, 8-hour dark lighting schedule on behavioral traits and performance in male broiler chickens. The Journal of Applied Poultry Research, 2010; 19(3): 263-273.

[21] Kristensen H H, Perry G C, Prescott N B, Ladewig J, Ersboll A K, Wathes C M. Leg health and performance of broiler chickens reared in different light environments. Br Poult Sci, 2006; 47(3): 257-263.

[22] Olanrewaju H A, Purswell J L, Collier S D, Branton S L. Effects of genetic strain and light intensity on blood physiological variables of broilers grown to heavy weights. Poult. Sci., 2014; 93(4): 970-978.

[23] Velo R, Ceular A. Effects of stocking density, light and perches on broiler growth. Anim. Sci. J., 2017; 88(2): 386-393.

[24] Yang Y, Pan C, Zhong R, Pan J. Artificial light and biological responses of broiler chickens: dose-response. J. Anim. Sci., 2018; 96(1): 98-107.

[25] Kristensen $\mathrm{H} \mathrm{H}$, Aerts J M, Leroy T, Wathes C M, Berckmans D. Modelling the dynamic activity of broiler chickens in response to step-wise changes in light intensity. Appl. Anim. Behav. Sci., 2006; 101(1): 125-143.

[26] Lien R J, Hess J B, McKee S R, Bilgili S F. Effect of light intensity on live performance and processing characteristics of broilers. Poult. Sci., 2008; 87(5): 853-857.

[27] Kristensen H H, Prescott N B, Perry G C, Ladewig J, Ersbøll A K, Overvad $\mathrm{K} \mathrm{C}$, et al. The behaviour of broiler chickens in different light sources and illuminances. Appl. Anim. Behav. Sci., 2007; 103(1-2): 75-89.

[28] Pittet F, Coignard M, Houdelier C, Richard-Yris M A, Lumineau S. Effects of maternal experience on fearfulness and maternal behaviour in a precocial bird. Anim. Behav., 2013; 85(4): 797-805.

[29] Badas E P, Martinez J, Rivero-de Aguilar J, Ponce C, Stevens M, Merino S. Colour change in a structural ornament is related to individual quality, parasites and mating patterns in the blue tit. The Science of Nature, 2018; 105: 17.

[30] Morinay J, Forsman J T, Kivelä S M, Gustafsson L, Doligez B Heterospecific nest site copying behavior in a wild bird: assessing the influence of genetics and past experience on a joint breeding phenotype. Frontiers in Ecology and Evolution, 2018; 5: 167.

[31] Nocera J J, Ratcliffe L M. Migrant and resident birds adjust antipredator behavior in response to social information accuracy. Behav. Ecol., 2010; 21(1): 121-128. 